Bilingualism: Language and Cognition 22 (4), 2019, 836-847 (C) Cambridge University Press 2018 This is an Open Access article distributed under the terms of the Creative Commons Attribution licence (http://creativecommons.org/licenses/by/4.0/), which permits unrestricted re-use, distribution, and reproduction in any medium, provided the original work is properly cited. doi:10.1017/S1366728918000652

\title{
Having a different pointing of view about the future: The effect of signs on co-speech gestures about time in Mandarin-CSL bimodal bilinguals*
}

\author{
YAN GU \\ Tilburg center for Cognition and Communication, Tilburg \\ University, the Netherlands \\ Department of Experimental Psychology, University College \\ London, UK \\ YEQIU ZHENG \\ Department of Econometrics and Operations Research, Tilburg \\ University, the Netherlands \\ MARC SWERTS \\ Tilburg center for Cognition and Communication, Tilburg \\ University, the Netherlands
}

(Received: December 5, 2017; final revision received: May 8, 2018; accepted: May 15, 2018)

\begin{abstract}
Mandarin speakers often use GESTURES to represent time laterally, vertically, and sagittally. Chinese Sign Language (CSL) users also exploit SIGNS for that purpose, and can differ from the gestures of Mandarin speakers in their choices of axes and direction of sagittal movements. The effects of sign language on co-speech gestures about time were investigated by comparing spontaneous temporal gestures of late bimodal bilinguals (Mandarin learners of CSL) and non-signing Mandarin speakers. Spontaneous gestures were elicited via a wordlist definition task. In addition to effects of temporal words on temporal gestures, results showed significant effects of sign. Compared with non-signers, late bimodal bilinguals (1) produced more sagittal but fewer lateral temporal gestures; and (2) exhibited a different temporal orientation of sagittal gestures, as they were more likely to gesture past events to their back. In conclusion, bodily experience of sign language can not only impact the nature of co-speech gestures, but also spatio-motoric thinking and abstract space-time mappings.
\end{abstract}

keywords: temporal gesture, bimodal bilingual, space-time metaphor, Chinese Sign Language, sign on gesture, Mandarin Chinese

\section{Introduction}

People use space to represent the abstract concept of time (e.g., Casasanto \& Boroditsky, 2008; see reviews of Bender \& Beller, 2014; Núñez \& Cooperrider, 2013). For instance, we often talk about time in terms of space such as in phrases like: "The future is lying ahead; the past is behind us" (Lakoff \& Johnson, 1980). In addition, humans also tend to gesture to visually express time in space. English people may refer to the future by pointing to the front of their body and indicate the past by pointing to their back (also left-right for past-future) (Casasanto \& Jasmin, 2012; Cooperrider \& Núñez, 2009; Walker

* The first author received financial support from The Netherlands Organization for Scientific Research, via NWO Promoties in de Geesteswetenschappen (322-89-007) and the second author received financial support from NWO Research Talent (406-15-298), which are gratefully acknowledged. We thank Yufen Chi, Weidong Zheng, and Qingshi Zhu who have assisted us in running experiments. We are grateful to Marieke Hoetjes, Rein Cozijn, editor Kira Gor and the three anonymous reviewers for their comments on the manuscript, and Arthur van Soest for his advice on statistics. Preliminary results of this study have been presented at the $7^{\text {th }}$ Conference of International Society for Gesture Studies in Paris (ISGS 2016), and "The role of gesture in cognitive and linguistic processes" workshop at the University of Warwick (July, 2017).
\& Cooperrider, 2016). Such TEMPORAL GESTURES with the future-in-front and the past-at-back mappings sound common for many Westerners.

However, across cultures and languages, people may gesture about time vastly differently. Take the temporal gestures of FUTURE: For instance, residents of Pormpuraaw (Australia) point the future to the front of them only when they are facing the west, because they always arrange temporal order according to cardinal directions from east to west (Boroditsky \& Gaby, 2010). Interestingly, Aymara speakers (South America) point the future to their back as they believe that the future is unseen/unknown (Núñez \& Sweetser, 2006). Moroccans (North Africa) also have a strong tendency to gesturally position the past in the front and the future at their back, which is claimed to be shaped by their cultural attitude towards time, as Moroccans focus on past times and place high value on tradition (people who are past-focused metaphorically should have a tendency to place the past in front of them, "in the location where they could focus on the past literally with their eyes if past events were physical objects that could be seen" (de la Fuente, Santiago, Román, Dumitrache \& Casasanto, 2014, p.1684). Additionally, some Chinese people spontaneously direct their gestures to their front

Address for correspondence:

Yan Gu, Department of Experimental Psychology, Psychology and Language Sciences, Faculty of Brain Sciences, University College London, 26 Bedford Way, London WC1H 0AP, United Kingdom

Yan.gu@ucl.ac.uk 
when referring to past events, but the extent to which they perform past-in-front gestures is influenced by the accompanying temporal words (Gu, Zheng \& Swerts, in revision). Furthermore, people can even gesture about the future in some other space dimensions such as DOWNWARDS, or UPHILL, etc. (Gu, Mol, Hoetjes \& Swerts, 2017; Núñez, Cooperrider, Doan \& Wassmann, 2012).

Despite the fact that there are an increasing number of studies on the relation between speakers' gestures and their spatialisation of time (e.g., Bostan, Börütecene, Özcan \& Göksun, 2016; Floyd, 2016; Kita, Danziger \& Stolz, 2001; Le Guen \& Balam; 2012), we still have an incomplete understanding of why some communities gesture the future to the front whereas others gesture the past to the front. In the research reported here, we investigated this question by exploring the effect of temporal signs on temporal gestures in BIMODAL BILINGUALS, who know both a spoken language and a signed language (Emmorey, Boorin, Thompson \& Gollan, 2008). To the best of our knowledge, no study has researched the temporal gestures by people who have experience of sign language, which, in the case of Chinese, would represent an interesting group, as Mandarin Chinese-Chinese Sign Language (CSL) ${ }^{1}$ bimodal bilinguals share a similar culture as non-signers, but have acquired CSL which exploits different time-space mappings than Mandarin (see review below). Particularly, we are interested in whether Mandarin-CSL bimodal bilinguals gesture differently about time than Mandarin speakers who do not know CSL.

\section{Background}

\section{Mandarin speakers' temporal gestures}

It has been shown that Mandarin speakers make gestures on different axes in space to represent time. First, similar to English speakers, Chinese people most often produce lateral temporal gestures, with the past on the left and the future on the right side. However, different from most

1 The sign languages used in mainland China are generally called Chinese Sign Language (CSL) (Fischer \& Gong, 2010). CSL has different dialects such as the northern (Beijing) CSL and the southern (Shanghai) CSL, which sometimes can even be mutually unintelligible (Yang, 2005). The China Association of the Deaf has been making efforts to unify and standardise CSL since the late 1950s. An authorized dictionary, Chinese Sign Language (zhōng-guó shǒu-yǔ) (China Association of the Deaf and Hard of Hearing, 2003) is used in China to standardise CSL. The dictionary has collected the signed forms of more than 5,000 Chinese words that are common in use in both Beijing and Shanghai (representing the northern and the southern varieties). This standard variety is learned by users of both dialects and it is now widely used in education, on television and by interpreters (Yang, 2015). Note that we will deal with only one variety, namely the Standard CSL, which was the language taught to the hearing L2 learners in our study.
Table 1. Examples of future-in-front/past-at-back and past-in-front/future-at-back mappings in Mandarin.

\begin{tabular}{|c|c|c|c|c|}
\hline $\begin{array}{l}\text { Example } \\
\text { (1) }\end{array}$ & $\begin{array}{l}\text { 展/zhăn } \\
\text { unfold }\end{array}$ & $\begin{array}{l}\text { 望/wàng } \\
\text { gaze-into-distance }\end{array}$ & $\begin{array}{l}\text { 未/wèi } \\
\text { hasn’t }\end{array}$ & $\begin{array}{l}\text { 来/lái } \\
\text { come }\end{array}$ \\
\hline & $\begin{array}{l}\text { Looking far } \\
\text { 回/huí } \\
\text { turn-around } \\
\text { Looking bac }\end{array}$ & $\begin{array}{l}\text { ahead/into the future } \\
\text { 首/shǒu } \\
\text { head } \\
\mathrm{k} \text { to the past. }\end{array}$ & 过/guò & $\begin{array}{l}\text { 去/qù } \\
\text { go }\end{array}$ \\
\hline $\begin{array}{l}\text { Example } \\
\text { (2) }\end{array}$ & $\begin{array}{l}\text { 前/qián } \\
\text { front } \\
\text { the day befo }\end{array}$ & $\begin{array}{l}\text { 天/tiān, } \\
\text { day, } \\
\text { e yesterday, }\end{array}$ & $\begin{array}{l}\text { 今/jīn } \\
\text { today } \\
\text { from no }\end{array}$ & $\begin{array}{l}\text { 后/hòu } \\
\text { back } \\
\text { W on }\end{array}$ \\
\hline
\end{tabular}

Westerners, Chinese can exploit a vertical axis as well to gesture about time, as they tend to spontaneously point UPWARDS for the time conception of "last week" and DOWNWARDS for "next week" (Gu et al., 2017).

Additionally, Mandarin speakers can perform sagittal gestures to express time. On the one hand, they can point the future to the front of their body and the past to the back, which is in line with the Mandarin future-in-front/past-atback sagittal space-time metaphors (Table 1, Example 1). On the other hand, a Mandarin speaker can point to the front of his/her body to refer to the conception of temporal "before" (Chui, 2011). Recent research also reveals that past-in-front gestures were more often associated with past-in-front/future-at-back space-time metaphors. As Example (2) shows, the sagittal words for spatial "front" (前/qián)" and "back" (后/hòu) are also used as temporal conceptions of "before/past" and "after/future". Such sagittal spatial metaphors for time suggest past-infront/future-at-back space-time mappings, and, therefore, may significantly influence the direction of sagittal temporal gestures. Partially due to this lexical effect, some Mandarin speakers even explicitly report to believe the future to be positioned behind and the past in front of them (i.e., past-in-front space-time mappings) (Gu et al., in revision).

\section{Temporal signs in CSL}

CSL users also make use of the lateral, vertical, and sagittal spatial representations to express the conception of time. In many sign languages, the lateral axis is often used to express a sequence timeline, which is parallel to the signers' body and extends from left to right, representing earlier to later time periods (e.g., Nilsson, 2016; Wilcox, 2002). It is used when signers refer to ordered events that are unrelated to the utterance time (Emmorey, 2001). Zheng (2009) finds that users of CSL are consistent in listing events that happened at a different time from the left to the right.

As for the vertical timeline, CSL signers make use of vertical spatial metaphors of "up" and "down" to represent 
time conceptions of "early" and "late", or the sequence of events. For instance, the temporal conception of "future" can be signed "downwards" (Wu \& Li, 2012; Zheng, 2009).

Furthermore, the sagittal axis is often used for what could be termed a deictic timeline. Similar to other sign languages in the world (e.g., Cabeza Pereiro \& Fernández Soneira, 2004; Maeder \& Loncke, 1996; Schermer \& Koolhof, 1990, see a review in Sinte, 2013), CSL signers' bodies are often referred to as a deictic reference point of the timeline, such that locations near the signers are often used for "now", and the future is signed more to their front and the past to their back (Wu \& Li, 2012; Zheng, 2009).

\section{Time in hands: gestures vs. signs}

Interestingly, there are dramatic DIFFERENCES in the deictic sagittal timelines between CSL and Mandarin Chinese. As stated above, Mandarin Chinese contains space-time metaphors that suggest both future-infront/past-at-back and past-in-front/future-at-back spacetime mappings. Accordingly, Mandarin speakers can not only produce future-in-front/past-at-back temporal gestures, but also past-in-front/future-at-back gestures. However, the sagittal lexical signs of CSL do not show this variation, as they represent ONLY future-infront/past-at-back space-time mappings, in this way being different from Mandarin Chinese. For instance, the time conceptions of "the day before yesterday" and "the day after tomorrow" in Mandarin are expressed in a completely reversed manner from what is the case in CSL (Wu \& Li, 2012; Zheng, 2009). That is, in Mandarin the direction of "the day before yesterday (前天/qiántiān, front day)" is literally to the front, and "the day after tomorrow (后天/hòu-tiān, back day)" is literally to the back, which is often reflected in the directionality of the co-speech sagittal temporal gestures by Mandarin speakers (Gu et al., in revision). By contrast, in CSL the temporal sign of "the day before yesterday" is signed to the back, whereas the temporal sign of "the day after tomorrow" is signed to the front (Zheng, 2009).

Additionally, although Mandarin speakers and CSL signers both use 3D manual movements to indicate time, the relative proportion of the three time axes may be different, since Mandarin speakers predominantly produce temporal gestures on the lateral axis ( $\mathrm{Gu}$ et al., 2017) whereas an empirical survey showed that CSL deaf signers mostly produce temporal signs on the sagittal and vertical axes (Zheng, 2009).

\section{Do speakers' gestures change after learning a spoken or signed language?}

There has been a long interest on whether speakers gesture differently after learning an L2, even when the existing studies provide mixed results (e.g., Brown \& Gullberg, 2008; Casey \& Emmorey, 2009; Özçalişkan, 2016; Pika, Nicoladis \& Marentette, 2006). For unimodal (nonbimodal) bilinguals, Brown and Gullberg (2008) found that there were influences of an L2 on co-speech gestures of an L1. For instance, it was found that intermediate Japanese learners of English gestured slightly differently in their L1 Japanese than Japanese monolinguals when talking about motion events. Specifically, JapaneseEnglish speakers (similar to English monolinguals) were less likely to perform a gesture that expressed manner of motion than monolingual Japanese, while their speech conveyed manner information. By contrast, Choi and Lantolf (2008) found that even advanced English learners of Korean as an L2 or Korean learners of an L2 English still retained their L1 co-speech gesture patterns when expressing manner of motion in their L1 language. Similarly, Özçalişkan (2016) found that Turkish-English bilinguals still followed L1 co-speech gesture patterns even when speaking L2.

As for bimodal bilinguals, the very few studies about their gestures reveal that there is probably an influence of a signed language on the co-speech gesture patterns in a first spoken language. For instance, an L2 sign language may affect the production of co-speech gestures or facial expressions when bimodal bilinguals speak in their L1 (Pyers \& Emmorey, 2008). Additionally, two studies have shown that American Sign Language (ASL)English bilinguals may have a higher co-speech iconic gesture rate than English non-signers (Casey \& Emmorey, 2009; Casey, Emmorey \& Larrabee, 2012). These results seem to suggest that gestures and signs stem from the same manual articulation system, and that there is an interaction between a signed language production system and the cospeech gesture production system (Brentari, Nadolske \& Wolford, 2012; Emmorey et al., 2008).

However, the studies on gestures discussed above, regardless of whether they were dealing with unimodal or bimodal bilinguals, predominately have focused on how gestures for motion events or gesture frequency and form can be affected by knowing a second spoken/signed language. No studies have looked into how the CONTENT of gestures (e.g., the abstract concept of space-time mappings represented in gestures) can be affected by the experience of a signed language.

\section{The current study}

The current study aims to investigate whether the experience of CSL influences the production of cospeech gestures about time in bimodal bilinguals. We will explore firstly whether Mandarin-CSL bimodal bilinguals perform different patterns of temporal gesture from Mandarin speakers, in terms of the relative proportion of three axes. Second, focusing on the temporal orientation 
on the sagittal axis, we aim to find out whether hearing people who have learned CSL have a different direction of sagittal temporal gestures than Mandarin non-signers.

If it is the case (in line with previous studies) that the gesture production and sign production systems are interconnected (e.g., Emmorey et al., 2008) in a way that bimodal bilinguals are accustomed to perform manual movements in certain axes or directions, given the differences between temporal gestures and signs, we predict that bimodal bilinguals will have more sagittal and vertical temporal gestures but fewer lateral temporal gestures than Mandarin speakers who are non-signers. Additionally, Mandarin-CSL bilinguals are less likely to perform past-in-front gestural mappings than Mandarin speaking non-signers.

Furthermore, given that spontaneous gestures are a window into people's spatio-temporal thinking (e.g., Casasanto \& Jasmin, 2012; Cienki, 1998; Núñez \& Sweetser, 2006), providing a "vivid and naturalistic source of evidence for the use of space in abstract reasoning" (visualising thought) (Cooperrider, Gentner \& Goldin-Meadow, 2016; Cooperrider \& Goldin-Meadow, 2017; Tversky, 2011), the study of co-speech temporal gestures by late bimodal bilinguals may reveal the effect of cross-modal spatial metaphors of time on people's mental space-time mappings. Thus this study can show the cross-linguistic influence of an L2 on an L1 and may further help clarify the problem of the restructuring of temporal conceptualisation after learning an L2.

\section{Method}

\section{Participants}

Forty-four participants, including 10 hearing MandarinCSL late bimodal bilinguals (6 female; $M_{\text {age }}=39.2 \mathrm{yrs}$, $S D=7.7 \mathrm{yrs})$ and 34 Mandarin-speaking non-signers ${ }^{2}$ (22 females; $M_{\text {age }}=33.79 \mathrm{yrs}, S D=7.58 \mathrm{yrs}$ ), took part in the experiment in Rizhao, China. Three Mandarinspeaking non-signers were excluded from the analyses, as they did not produce any gestures.

All late bimodal bilinguals were born into hearing families and acquired standard CSL as a second language later in their life (average age of acquisition $=20.6 \mathrm{yrs}$, $S D=3.3 \mathrm{yrs}$ ). They were fluent users of standard CSL with an average of 18.6 years of signing experience $(S D$ $=9.2)$. Their CSL proficiency levels $(M=8.6, S D=$ 1.07, 10-point scales) were assessed by a CSL teacher from a school for special education. This assessment was done after all the participants had finished the

2 The monolingual data were from a corpus collected in Gu, Zheng \& Swerts (in revision)'s study. We used their original data to do different analyses so that they could be used as comparison materials in this study. experiment so that participants would not infer a focus on manual movements in the study. These bimodal bilinguals were teachers of deaf children, and none of them were interpreters.

Additionally, the English proficiency levels of participants of both groups were minimum $(M=1.36$, $S D=.66,1=$ hardly know any English; $2=$ beginner to lower-intermediate), as reported on a 5-point-scales' self-assessment.

\section{Materials and procedure}

A word definition task was used to elicit participants' spontaneous gestures, inspired by previous studies (e.g., Gu et al., 2017; Núñez et al., 2012) that showed the effectiveness of this method. We constructed twelve wordlists, which consisted of five wordlists of time conceptions and seven of fillers. Each wordlist had two to four expressions that were thematically related (e.g., "yesterday", "today", and "tomorrow"). In total, there were thirteen Mandarin temporal expressions (see Appendix).

The experiment was ostensibly set up as a study of speakers' short-term memory and addressees' long-term memory. All bimodal bilinguals and non-signers took the role of speakers to fulfil the word definition task in their native language: that is, spoken Mandarin (not in sign language). All participants were told that the task was in Mandarin and the addressees could only speak Mandarin Chinese. They were asked to remember each wordlist shortly after seeing it twice presented on screen. Then they had to tell and explain the words from each wordlist as explicitly as possible to Mandarin-speaking addressees who could ask them clarification questions (Fig. 1; for more details see the same method in Gu et al., 2017). The addressees were told to remember speakers' descriptions for a later memory test. However, the latter test actually did not take place as they were confederates. The experiment was videotaped after obtaining participants' written consent. Gestures or CSL were not mentioned at any moment and debriefing responses revealed that participants had not realised that the study was about speakers' gestures or manual movements.

\section{Gesture coding}

Co-speech temporal gestures were annotated in ELAN (Lausberg \& Sloetjes, 2009). A first coder did an initial coding, viewing the entire video with the audio. The axes of gestures were coded as vertical, lateral, or sagittal, with an indication of the directionality of each axis (Casasanto \& Jasmin, 2012; Gu et al., 2017). Additionally, although bimodal bilinguals were speaking to non-signers, they might still produce a small proportion of signs (e.g., about 3\%, Casey \& Emmorey, 2009). CSL temporal signs were noted when they were identifiable lexical signs, or hand movements that a non-signer would unlikely produce (Casey \& Emmorey, 2009; Casey et al., 2012). 


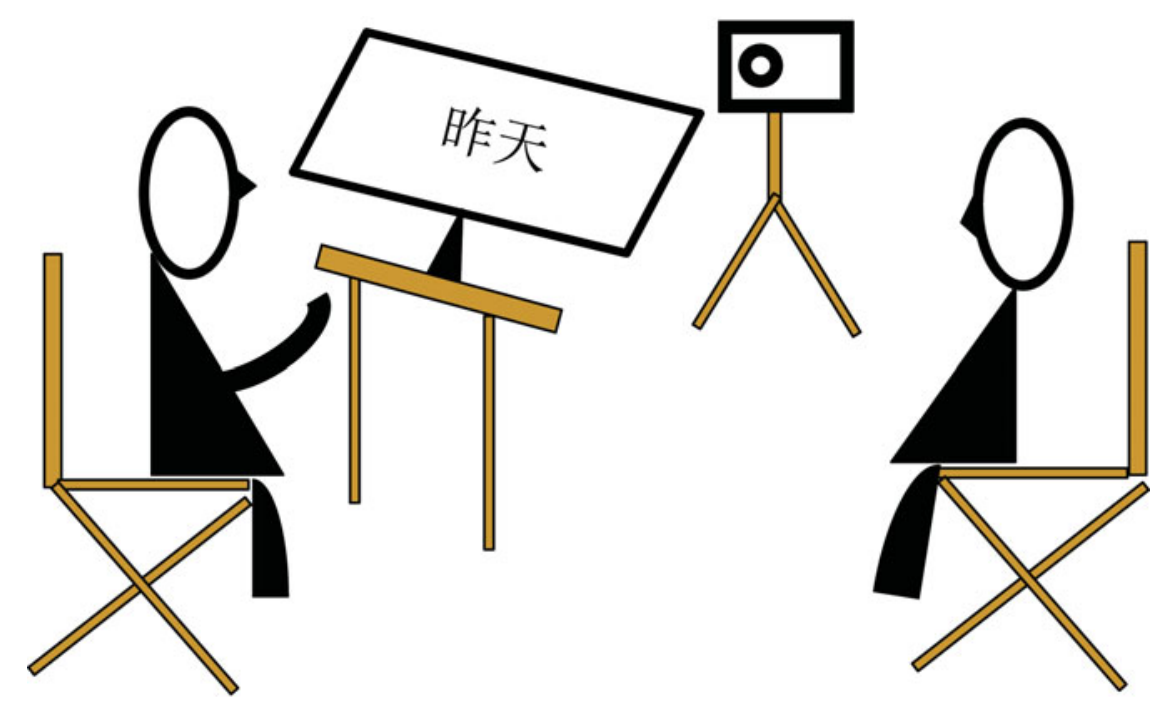

Figure 1. (Colour online) Schematic illustration of the experimental set-up.

Six CSL temporal signs (about 2.8\%) were detected and were excluded from the analyses (e.g., a temporal sign of "morning" was produced when the Mandarin word "morning" was uttered: that is, a movement of one hand starts with a palm down horizontally in front of the chest, with four fingers and thumb pinched, and the hand moves up slowly with fingers gradually opened, indicating the sky is lighting up).

Furthermore, the temporal words accompanying temporal gestures were transcribed. These could contain temporal words explicitly having vertical spatial references to "up" and "down" (e.g., 上周/shàng-zhōu, above week, "last week"), sagittal spatial references to “front” (前/qián) and “back” (后/hòu) (e.g., 前年/qiánnián, front year, "the year before last year"), or words without having such lexical cues (e.g., 昨天/zuó-tiān, "yesterday"). These temporal words were coded in three categories (vertical; sagittal; neutral).

In total, we obtained 719 temporal word-gesture tokens, including 212 from late bimodal bilinguals, and 507 from Chinese non-signers. The average number of gestures by bimodal bilinguals $(M=21.2)$ tended to be significantly higher than that of non-signers $(M=$ 14.9), $t=1.52, p=.067$ (one-tailed with a directional hypothesis). The pattern of increased gesture production for bimodal bilinguals compared to non-signers is in line with previous results for ASL-English bilinguals (Casey \& Emmorey, 2009; Casey et al., 2012).

The reliability of the annotation of the gestures was established by having $53 \%$ of the data coded by a naïve second coder. The two coders agreed on the gesture axes judgement on $92.31 \%$ of the tokens $(N=380)$, Cohen's Kappa $=0.87$ (referring to "Excellent" agreement). In cases of disagreement, the two coders discussed and reached agreement on the labels, and these consensus labels were used for the final analysis.

\section{Statistical analyses}

A mixed multinomial logit model for panel data was used (Croissant, 2012) to compare the gesture proportion of three axes, with GROUP (late bimodal bilinguals vs. Mandarin-speaking non-signers) as a main independent variable and TEMPORAL GESTURE AXIS $(\mathrm{L} ; \mathrm{V} ; \mathrm{S})$ as a dependent variable. We started with the maximal random effect structure, including random intercepts and random slopes for the crucial independent variable GROUP. However, the standard deviations of random slopes on GROUP were insignificant, so the random slopes were not used in the final model. Given that previous research has shown that temporal words can have an influence on gestures (e.g., Gu et al., 2017), we also controlled for the type of temporal words accompanying temporal gestures (vertical; sagittal; neutral). To compare the direction of sagittal temporal gestures, a binary logistic regression for panel data was used, with group as a main independent variable and the direction of sagittal gestures (past-in-front or future-in-front) as a dependent variable. Both models have taken individual differences into consideration and dealt with the repeated observations from the same individuals.

\section{Results and analyses}

\section{Temporal gestures on the lateral, vertical, and sagittal axes}

As Fig. 2 shows, late bimodal bilinguals displayed a different distribution of temporal gestures on the three axes than the non-signers. Specifically, non-signers performed $48.72 \%$ of the temporal gestures on the lateral 


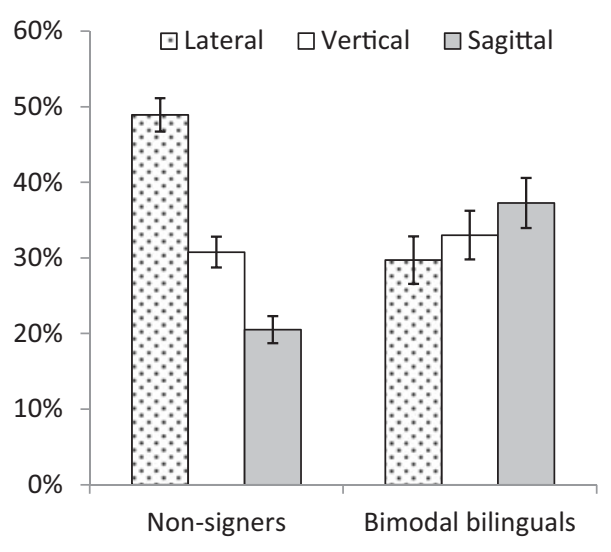

Figure 2. Distribution of temporal gestures on the three axes by late bimodal bilinguals and non-signers. Error bars show standard errors of the mean.

axis whereas late bimodal bilinguals performed only $29.72 \%$ of gestures on the lateral axis. Instead, late bimodal bilinguals performed $37.26 \%$ of the temporal gestures on the sagittal axis and $33.02 \%$ on the vertical axis, which, respectively, was $16.75 \%$ and $2.25 \%$ more than those of non-signers.

A mixed multinomial logit regression $(N=719)$ of gesture axes on group (baseline: vertical axis) showed that late bimodal bilinguals were significantly less likely to perform lateral temporal gestures $(t=-2.42, p=.016$, $\beta=-0.74)$ but more likely to perform sagittal ones $(t=$ $4.13, p<.001, \beta=1.98)$ than the non-signers, controlling for the type of temporal words (vertical; sagittal; neutral) and age. The different distribution of axes between the two groups indicated that the production of temporal gestures can be influenced by the experience of learning temporal signs in CSL.

Additionally, as for the influence of the type of temporal words on temporal gestures, we found that participants were more likely to perform vertical temporal gestures when uttering vertical spatial metaphors for time than when uttering neutral temporal words, regardless of whether they were signers or non-signers, in that they would perform fewer lateral $(t=-10.21, p<.001$, $\beta=-2.89)$ or sagittal temporal gestures $(t=-9.66$, $p<.001, \beta=-4.97)$, controlling for group and age. Similarly, participants were more likely to produce sagittal temporal gestures when uttering sagittal spatial metaphors for time than when uttering neutral temporal words $(t=$ $2.26, p=.024, \beta=.87)$. The results indicated that the concurrent temporal words also had an effect on the choice of temporal gesture axes.

\section{Directionality of sagittal temporal gestures}

Focusing on the directionality of sagittal temporal gestures, non-signers performed about $49.04 \%$ of the sagittal temporal gestures with the past to the front and the

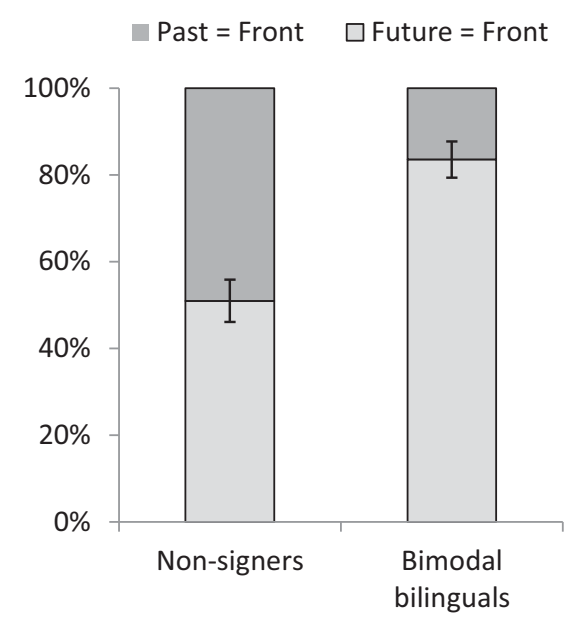

Figure 3. Orientation of sagittal temporal gestures by late bimodal bilinguals and non-signers. Error bars show standard errors of the mean.

future to their back (past-in-front/future-at-back gestures) and $50.96 \%$ with the future to the front and the past to the back (future-in-front/past-at-back gestures). However, the proportion of past-in-front/future-at-back gestures by late bimodal bilinguals was only $16.46 \%$, and the proportion of future-in-front/past-at-back gestures was $83.54 \%$ (Fig. 3).

A binary logistic regression $(N=183)$ of sagittal temporal gesture direction on group showed that late bimodal bilinguals performed a significantly lower proportion of past-in-front/future-at-back temporal gestures than the non-signers, Wald $\chi^{2}(1)=5.12, p=$ $.024, \beta=-6.85,95 \% \mathrm{CI}=[-12.78,-.92]$, even after controlling for the type of temporal words co-occurring with gestures (vertical; sagittal; neutral). This indicated that after learning CSL, late bimodal bilinguals were more likely to have a future-in-front/past-at-back temporal orientation as visible in their sagittal temporal gestures.

Furthermore, it has been claimed that Mandarin speakers' past-in-front temporal gestures are predominately produced when speakers utter sagittal temporal words with past-in-front metaphors (e.g., qián-tiān/前天, front day, "the day before yesterday") (Gu et al., in revision; Lai \& Boroditsky, 2013). For instance, in this study, when Mandarin-speaking non-signers uttered past-infront metaphors, $72.34 \%$ of the sagittal temporal gestures were the past-in-front temporal gestures. However, in this case, the proportion by late bimodal bilinguals was only $22.22 \%$ (Figure 4 ), which was significantly smaller (Wald $\chi^{2}(1)=54.16, N=83, p<.001, \beta=-19.75,95 \%$ $\mathrm{CI}=[-25.01,-14.49])$, and the majority of sagittal temporal gestures were instead produced according to the future-in-front mapping (77.78\%). Thus, late bimodal bilinguals had a different direction of sagittal gestures than non-signers even when both groups were uttering the same overt past-in-front space-time metaphors. The 


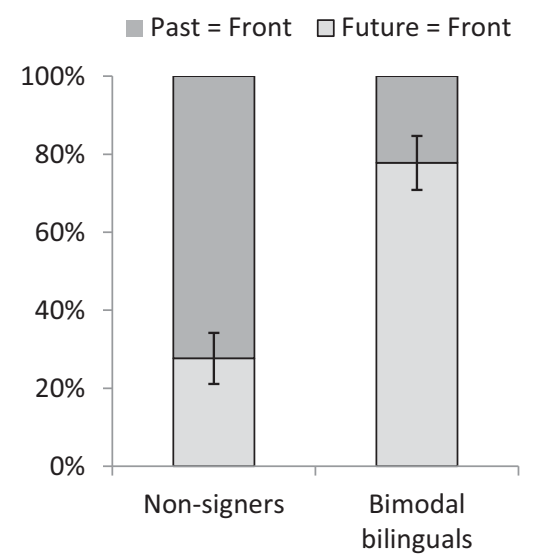

Figure 4. Orientation of sagittal temporal gestures accompanied by past-in-front temporal sagittal words. Error bars show standard errors of the mean.

results indicated that the experience of temporal signs influenced temporal gestures.

\section{Discussion}

This study is the first that explored temporal gestures by bimodal bilinguals, and the first to look into effects of temporal signs on temporal gestures. Our results have shown that both Mandarin-speaking non-signers and Mandarin-CSL late bimodal bilinguals could perform spontaneous temporal gestures at the lateral, vertical, and sagittal axes. However, the two groups were significantly different in their use of temporal gestures on the three axes, as well as in their direction of sagittal temporal gestures. Although the results of this study were admittedly obtained based on a relative small number of bimodal bilinguals, these findings have a number of important theoretical implications.

First, our findings support the claim that there is an interconnection between the co-speech gesture production system and a sign language production system (Emmorey et al., 2008). The few studies on this topic have mainly focused on the changes in gesture rate, character viewpoint, and handshape after learning American Sign Language (ASL). Although it has been observed in these studies that there was an increase of gesture rate in ASL learners, sometimes "these changes were not large enough to create significant group differences" in comparison to non-signers (Casey et al., 2012) (Note in that study ASL learners had only one-year of ASL instruction). The present study, however, focusing on the study of temporal gestures, provides additional evidence that the knowledge and experience of an L2 sign language can indeed impact the content and form of $\mathrm{L} 1$ co-speech gestures.

Second, these results point out that there may be crosslinguistic influences of the L2 on the L1 (e.g., Brown \& Gullberg, 2008, 2011; Zou, Abutalebi, Zinszer, Yan, Shu, Peng \& Ding, 2012). Studies have shown that languages are co-activated in a bilingual mind (e.g., Van Hell \& Dijkstra, 2002). For instance, there is an unconscious access to the sound form of Chinese words when ChineseEnglish bilinguals read or listen to English words (Wu \& Thierry, 2010). Such cross-language interactions can even occur across modalities (e.g., Emmorey, Grabowski, McCullough, Ponto, Hichwa \& Damasio, 2005; Giezen \& Emmorey, 2016; Ortega \& Morgan, 2015). For example, Morford, Wilkinson, Villwock, Piñar and Kroll (2011) found that ASL-English bilingual deaf readers activate the ASL translations of written words in English even when the task does not explicitly require the use of ASL. Recent ERP research also reveals that there is an implicit co-activation of ASL in deaf readers (Meade, Midgley, Sevcikova Sehyr, Holcomb \& Emmorey, 2017). In our study, late bimodal bilinguals produced significantly more sagittal temporal gestures than non-signers. Given that CSL mostly makes use of the sagittal spatial metaphors for time (Zheng, 2009), a speculative explanation for the result can be that even when Mandarin is the target language for production, the detailed spatial information for temporal expressions in CSL is still activated, which may prime the action production system that generates temporal gestures (Casey \& Emmorey, 2009).

One possible concern is that these manual movements produced by bimodal bilinguals were not co-speech gestures but CSL signs. This is quite unlikely because even native bimodal bilinguals only produce very few signs when interacting with non-signers (e.g., only $3 \%$, Casey \& Emmorey, 2009), and in our study participants were late bimodal bilinguals and their signs have been excluded in the analyses. Additionally, it was also visible by the number of fingers in the gestures. For example, the concept of "the day before yesterday" in CSL is expressed by the use of the index and middle fingers to point to the back once, whereas the gestures we obtained did not show such a pattern.

Furthermore, our results also suggest that the acquisition of a signed language may have an impact beyond the nature of gestures that accompany the native spoken language (cf. Casey et al., 2012; Emmorey, Giezen \& Gollan, 2016). For instance, an intriguing result is that Mandarin-CSL late bilinguals were highly unlikely to perform past-in-front/future-at-back temporal gestures as opposed to Chinese non-signers who would often do so. In other words, the future-in-front/past-at-back mapping was activated to a greater extent in bimodal bilinguals than in Mandarin non-signers. Strikingly, even when the sagittal temporal gestures were accompanied by the sagittal past-in-front words, a situation in which the gesture direction would most likely be influenced by the uttering of such overt words, late bimodal bilinguals still rarely directed the past to their front. If spontaneous gestures are a visible embodiment of cognition (Alibali, 2005; Hostetter \& Alibali, 2008) which provide a window 
into people's mental space-time mapping (e.g., Casasanto \& Jasmin, 2012; Cienki, 1998; Núñez \& Sweetser, 2006; Walker \& Cooperrider, 2016), it is likely that learning CSL changes Mandarin speakers' conceptualisations of space-time mappings.

Such differences in sagittal space-time mappings may be explained in terms of differences in time perspectivetaking $^{3}$, related to two possible systems of space-time metaphor in language. There are two types of time perspectives, i.e., moving-ego and moving-time (e.g., Moore, 2011; Núñez, Motz \& Teuscher, 2006; Walker, Bergen \& Núñez, 2017). When a person takes an egomoving perspective, $\mathrm{s} /$ he moves forward in the timeline, from past to future, e.g., "We look forward to the future ahead". When that person takes a time-moving perspective (e.g., "Christmas is coming"), s/he still faces the future, but time is conceived of as a river or conveyor belt on which events are moving from the future to the past (Gentner, Imai \& Boroditsky, 2002). In this perspective, the FRONT of a timeline can be assigned to a past (earlier) event (e.g., in the timeline May is before (in front of) June).

According to previous studies, English speakers usually take an ego-moving perspective, whereas Mandarin speakers mostly take a time-moving perspective (e.g., Gentner et al., 2002; Xiao, Zhao \& Chen, 2017; Yu, 2012). For example, Mandarin-English speakers were influenced by the English time perspective even when they were speaking Mandarin, such that Mandarin-English speakers were less likely to take a time-moving perspective than Mandarin monolinguals (Lai \& Boroditsky, 2013). Similarly, given that signers of CSL mainly take the egomoving time perspective (the deictic of time in CSL is moving ego, Wu \& Li, 2012), late bimodal bilinguals may be influenced by the CSL time perspective even in a nonsigning context.

One may further ascribe such differences in spatiotemporal reasoning to the different uses of spatial metaphors for time between Mandarin Chinese and CSL, given that Mandarin Chinese contains both lexicon words suggesting future-in-front/past-at-back and pastin-front/future-at-back space-time mappings, whereas the sagittal lexical signs of CSL do not show this variation as they represent only future-in-front/past-at-back spacetime mappings ( $\mathrm{Wu} \& \mathrm{Li}, 2012$; Zheng, 2009). For instance, a recent study has shown that Chinese deaf signers display a different spatio-temporal reasoning than

\footnotetext{
3 One reviewer pointed out that the cross-linguistic differences in timespace mappings are unlikely raised from the moving-ego vs. movingtime perspectives. When an ego is involved in the timeline, in both perspectives, the future is ahead and the past is behind; neither presents a scenario in which the past is ahead (because this would be characterised by a reverse moving time perspective, in which time moves from behind forward). More studies are needed to better understand the psychological realisation of Chinese sagittal spacetime mappings.
}

Mandarin speakers. Specifically, participants were asked to fulfil a Mandarin temporal performance task, in which they had to label the Mandarin past and the future concepts in front-back space. The results revealed that CSL deaf signers with higher Mandarin proficiency were more likely to perform past-in-front/future-at-back space-time mappings than signers with lower Mandarin proficiency (Gu, Zheng \& Swerts, 2017).

Given that a body of evidence has shown that space-time metaphors can influence people's mental representation of time (e.g., Boroditsky, 2000, 2001; Bylund \& Athanasopoulos, 2017; Hendricks \& Boroditsky, 2017), it is plausible that the learning of cross-modal spatio-temporal metaphors of CSL can also impact learners' time conceptualisations. For instance, Mandarin-CSL bilinguals "learn" to reconstruct the sagittal mental space-time mappings with the "future-infront/past-at-back" as the dominant mappings.

Then the question is raised as to whether the differences in sagittal space-time mappings between Mandarin speakers and late bimodal bilinguals were merely due to bimodal bilinguals' learning of an L2 (having a different space-time metaphor than Mandarin). If this were the case, we would expect that Mandarin-English bilinguals may also have a similar change in space-time mappings as revealed by their co-speech gestures (since English, like CSL, usually also does not use past-in-front mappings). However, previous studies did not show such a pattern (e.g., Fuhrman, McCormick, Chen, Jiang, Shu, Mao \& Boroditsky, 2011; Gu, Hoetjes \& Swerts, in preparation), so that one can raise the question why MandarinEnglish bilinguals still perform a large proportion of "past-in-front" temporal gestures while speaking Mandarin.

Apart from the possible influence of the L2 proficiency, this could be due to the fact that in English, these metaphoric gestures are not "learned" like CSL signs or emblematic gestures. Temporal conceptions are spatially more iconic in a signed language than a spoken language (e.g., CSL vs. English), as temporal signs are visually and physically salient in the signing movements. The acquisition of sign language requires the learner to linguistically make distinctions based on movement (Emmorey \& McCullough, 2009), and can enhance one's visual-spatial ability. For instance, habitual use of ASL may lead to enhanced memory for object orientation (Emmorey et al., 1998). Therefore, if one learns a sign pointing to the back for the conception of past and keeps on signing like this on and on, day in and day out, it is imaginable that the person can form a habitual mapping of the past to the back. This is also in line with the BODY-SPECIFICITY HYPOTHESIS (Casasanto, 2009) that particular patterns of bodily experience can give rise to corresponding habits of thinking, perceiving, and acting (Gibbs, 2003). 
Furthermore, signs can be regarded as a special kind of action, representing the world linguistically by use of space whereas gestures are also claimed to generate from the same process that generates actions (Chu \& Kita, 2016; Kita \& Özyürek, 2003). Gestures can be regarded as SimUlated ACTIONS (Hostetter \& Alibali, 2008), which have no physical consequence on the real world but share some properties with actions. Therefore, gesture and sign to some extent share the same action production system (e.g., Emmorey et al., 2008). The GestureFOR-CONCEPTUALISATION HYPOTHESIS proposes that performing actions or gestures can activate and change one's spatial thinking (Kita, Alibali \& Chu, 2017). We believe that signing, a special kind of action in space, may also activate and change one's spatial thinking. For example, when bimodal bilinguals are signing about abstract ideas (e.g., time), the spatial movements of their hands may activate different spatio-motoric information from that of non-signers, which may affect bimodal bilinguals' spatial thinking in the long run. Given that people use space to think about time (e.g., Casasanto \& Boroditsky, 2008), a different/new spatial thinking may consequently bring certain changes in space-time mappings, as shown in bimodal bilinguals' temporal gestures. Thus the results of this study appear to show an effect of (sign) language on thinking about time within a culture (Boroditsky, 2001; Gu et al., 2017).

Alternatively, the results could be explained by the possibility that signing in a manner consistent with a future-in-front/past-at-back frame of reference primes bimodal bilinguals to gesture in a similar manner. This possibility is consistent with the proposal of the Gesture as Simulated Action model (Hostetter \& Alibali, 2008): The activation of the motor system according to the spatio-temporal mapping via CSL may have primed the activation of the gesture system on a similar axis, which results in the effects observed in this study. This priming does not necessarily indicate that the bimodal bilingual's actual representations of time have changed, unlike the neo-Whorfian account that is mentioned above. (Nevertheless, this possibility of priming is harder to reconcile with the findings concerning the relationship between the directionality of gesture and verbal expressions) ${ }^{4}$.

Furthermore, one can even argue that co-speech temporal gestures do not necessarily reflect one's online conceptualisations of time, because they may only reveal speakers' implicit space-time mappings. Given all the above, future studies can use non-linguistic tasks (e.g., Fuhrman \& Boroditsky, 2010; Fuhrman et al., 2011) to further examine this in bimodal bilinguals of different signing proficiency.

\footnotetext{
4 We thank one anonymous reviewer for pointing out this alternative possibility.
}

Finally, the study provides a better understanding on the variation of the production of temporal gestures. Previous studies have shown that temporal gestures can be shaped by the reading and writing direction (e.g., Casasanto \& Jasmin, 2012; Cooperrider \& Núñez, 2009; Walker \& Cooperrider, 2016), linguistic space-time metaphors (Gu et al., 2017; Lai \& Boroditsky, 2013), cultural specific belief (Núñez \& Sweetser, 2006), use of cardinal frame of references (Boroditsky \& Gaby, 2010), and geographical environments (Núñez et al., 2012).

This study, on the one hand, showed that temporal gestures can be shaped by the accompanying words that happen to be uttered, e.g., vertical/sagittal temporal words can lead to more vertical/sagittal temporal gestures. On the other hand, with a comparison between MandarinCSL late bilinguals and Mandarin-speaking non-signers, we discovered that temporal gestures can be affected by people's bodily experience of sign language which may influence CSL users' spatio-temporal thinking. Note that such differences in gesture production were unlikely due to the lexical effect of Mandarin temporal words, because both groups were speaking in the same L1 and the differences still existed even when the uttered words were identical. Therefore, the different temporal gestures may be due to their different thinking of using the body to interact with the physical environment (spatio-motoric thinking, Kita, 2000) to represent time in space. Overall, all this evidence suggests that the ultimate production of temporal gestures is a result of the linguistic words and the metaphoric spatio-motoric thinking (cf. Kita \& Özyürek, 2003; Özçalişkan, 2016; Özçalişkan, Lucero \& GoldinMeadow, 2016).

\section{Conclusion}

In this study we examined whether the experience of CSL influences the production of co-speech gestures about time in late bimodal bilinguals. The results showed that hearing people who have learned CSL performed differently in temporal gesture production than Mandarin speakers, both in terms of relative proportion of three time axes, and of the temporal orientation of sagittal gestures. Based on the mechanism of a shared production system between gestures and signs (Emmorey et al., 2008), and the GESTURE-FOR-CONCEPTUALISATION HYPOTHESIS (Kita et al., 2017), we believe that the learning of a signed language can not only have an impact on the nature of cospeech gestures but may also exert an influence on users' spatio-motoric thinking and their abstract reasoning such as space-time mappings. Although the study of spacetime mappings in CSL has been somewhat neglected in the literature, this study could provide a first insight into a cross-modal influence of space-time metaphors on people's mental representations of time within a culture. Future research on this topic can adopt non-linguistic methods to corroborate our findings. 
Appendix. Wordlists of targeted time referents.

\begin{tabular}{|c|c|c|}
\hline & Chinese & Meaning \\
\hline (1) & $\begin{array}{l}\text { 上周, 下周 } \\
\text { shàng zhōu, xià zhōu }\end{array}$ & last week, next week \\
\hline (2) & $\begin{array}{l}\text { 昨天, 今天, 明天 } \\
\text { zuó tiān, jīn tiān, míng tiān }\end{array}$ & $\begin{array}{l}\text { yesterday, today, } \\
\text { tomorrow }\end{array}$ \\
\hline (3) & $\begin{array}{l}\text { 早晨, 晌午, 傍晚, 深夜 } \\
\text { zăo chén, shăng wǔ, bàng } \\
\text { wǎn, shēn yè }\end{array}$ & $\begin{array}{l}\text { morning, noon, evening, } \\
\text { late at night }\end{array}$ \\
\hline (4) & $\begin{array}{l}\text { 上辈子, 下辈子 } \\
\text { shàng bèi zi, xià bèi zi }\end{array}$ & previous life, next life \\
\hline (5) & $\begin{array}{l}\text { 前年, 后年 } \\
\text { qián nián, hòu nián }\end{array}$ & $\begin{array}{l}\text { the year before last year, } \\
\text { the year after next year }\end{array}$ \\
\hline
\end{tabular}

\section{References}

Alibali, M. W. (2005). Gesture in spatial cognition: Expressing, communicating, and thinking about spatial information. Spatial Cognition \& Computation, 5(4), 307-331. doi:10.1207/s15427633scc0504_2

Bender, A., \& Beller, S. (2014). Mapping spatial frames of reference onto time: A review of theoretical accounts and empirical findings. Cognition, 132(3), 342-382. doi:10.1016/j.cognition.2014.03.016

Boroditsky, L. (2000). Metaphoric structuring: Understanding time through spatial metaphors. Cognition, 75(1), 1-28. doi:10.1016/s0010-0277(99)00073-6

Boroditsky, L. (2001). Does language shape thought?: Mandarin and English speakers' conceptions of time. Cognitive Psychology, 43(1), 1-22. doi:10.1006/cogp.2001.0748

Boroditsky, L., \& Gaby, A. (2010). Remembrances of times East: Absolute spatial representations of time in an Australian aboriginal community. Psychological Science, 21(11), 1635-1639. doi:10.1177/0956797610386621

Bostan, İ., Börütecene, A., Özcan, O., \& Göksun, T., (2016). Temporal expressions in speech and gesture. In Papafragou, A., Grodner, D., Mirman, D., \& Trueswell, J.C. (Eds.). Proceedings of the 38th Annual Conference of the Cognitive Science Society (pp. 1871-1876). Austin, TX: Cognitive Science Society.

Brentari, D., Nadolske, M. A., \& Wolford, G. (2012). Can experience with co-speech gesture influence the prosody of a sign language? Sign language prosodic cues in bimodal bilinguals. Bilingualism: Language and Cognition, 15(2), 402-412. doi:10.1017/S1366728911000587

Brown, A., \& Gullberg, M. (2008). Bidirectional cross-linguistic influence in L1-L2 encoding of manner in speech and gesture. Studies in Second Language Acquisition, 30(2), 225-251. doi:10.1017/S0272263108080327

Brown, A., \& Gullberg, M. (2011). Bidirectional crosslinguistic influence in event conceptualization? Expressions of Path among Japanese learners of English. Bilingualism: Language and Cognition, 14(1), 79-94. doi:10.1017/S1366728910000064

Bylund, E., \& Athanasopoulos, P. (2017). The Whorfian time warp: Representing duration through the language hourglass. Journal of Experimental Psychology: General, 146(7), 911-916. doi: 10.1037/xge0000314

Cabeza Pereiro, C., \& Fernández Soneira, A. (2004). The expression of time in Spanish Sign Language (LSE). Sign Language \& Linguistics, 7(1), 63-82. doi:10.1075/s1l.7.1.06cab

Casasanto, D., \& Boroditsky, L. (2008). Time in the mind: Using space to think about time. Cognition, 106(2), 579-593. doi:10.1016/j.cognition.2007.03.004

Casasanto, D. (2009). Embodiment of abstract concepts: Good and bad in right- and left-handers. Journal of Experimental Psychology: General, 138(3), 351-367. doi:10.1037/a0015854

Casasanto, D., \& Jasmin, K. (2012). The hands of time: Temporal gestures in English speakers. Cognitive Linguistics, 23(4), 643-674. doi:10.1515/cog-2012-0020

Casey, S., \& Emmorey, K. (2009). Co-speech gesture in bimodal bilinguals. Language and Cognitive Process, 24(2), 290312. doi: 10.1080/01690960801916188

Casey, S., Emmorey, K., \& Larrabee, H. (2012). The effects of learning American Sign Language on co-speech gesture. Bilingualism: Language and Cognition, 15(4), 677-686. doi:10.1017/S1366728911000575P

China Association of the Deaf and Hard of Hearing (2003). Chinese Sign Language (中国手语). Beijing: Huaxia Publishing House.

Choi, S., \& Lantolf, J. P. (2008). Representation and embodiment of meaning in L2 communication: Motion events in the speech and gesture of advanced L2 Korean and L2 English speakers. Studies in Second Language Acquisition, 30(2), 191-224. doi:10.1017/S0272263108080315

Chu, M., \& Kita, S. (2016). Co-thought and cospeech gestures are generated by the same action generation process. Journal of Experimental Psychology: Learning, Memory, and Cognition, 42(2), 257-270. doi: $10.1037 / \mathrm{xlm} 0000168$

Chui, K. (2011). Conceptual metaphors in gesture. Cognitive Linguistics, 22(3), 437-458. doi:10.1515/cogl.2011.017

Cienki, A. (1998). Metaphoric gestures and some of their relations to verbal metaphorical expressions. In J. Koenig (Ed.), Discourse and cognition: Bridging the gap (pp. 189204). Stanford, CA: CSLI Productions.

Cooperrider, K., \& Núñez, R. (2009). Across time, across the body: Transversal temporal gestures. Gesture, 9(2), 181206. doi:10.1075/gest.9.2.02coo

Cooperrider, K., Gentner, D., \& Goldin-Meadow, S. (2016). Spatial analogies pervade complex relational reasoning: Evidence from spontaneous gestures. Cognitive Research: Principles and Implications, 1(1), 28. doi:10.1186/s41235-016-0024-5

Cooperrider, K., \& Goldin-Meadow, S. (2017). When gesture becomes analogy. Topics in Cognitive Science, 9(3), 719737. doi: 10.1111 /tops. 12276

Croissant, Y. (2012). Estimation of multinomial logit models in $\mathrm{R}$ : The mlogit Packages. $R$ package version 0.2-2. URL: http://cran.r-project.org/web/packages/mlogit/vignettes/ mlogit.pdf.

De la Fuente, J., Santiago, J., Román, A., Dumitrache, C., \& Casasanto, D. (2014). When you think about it, your past is in front of you: How culture shapes spatial 
conceptions of time. Psychological Science, 25(9), 16821690. doi:10.1177/0956797614534695

Emmorey, K., Klima, E., \& Hickok, G. (1998). Mental rotation within linguistic and non-linguistic domains in users of American Sign Language. Cognition, 68(3), 22146.

Emmorey, K. (2001). Space on hand: The exploitation of signing space to illustrate abstract thought. In M. Gattis (Ed.), Spatial schemas and abstract thought (pp. 147-174), Cambridge, MA, US: MIT Press.

Emmorey, K., Borinstein, H. B., Thompson, R., \& Gollan, T. H. (2008). Bimodal bilingualism. Bilingualism: Language and Cognition, 11(1), 4361. doi:10.1017/S1366728907003203

Emmorey, K., Grabowski, T., McCullough, S., Ponto, L. L., Hichwa, R. D., \& Damasio, H. (2005). The neural correlates of spatial language in English and American Sign Language: A PET study with hearing bilinguals. Neuroimage, 24(3), 832-840. doi: 10.1016/j.neuroimage.2004.10.008

Emmorey, K., \& McCullough, S. (2009). The bimodal bilingual brain: Effects of sign language experience. Brain and Language, 109(2-3), 124-32. doi:10.1016/j.band1.2008.03.005

Emmorey, K., Giezen, M. R., \& Gollan, T. H. (2016). Psycholinguistic, cognitive, and neural implications of bimodal bilingualism. Bilingualism: Language and Cognition, 19(2), 223-242. doi:10.1017/S1366728915000085

Fischer, S., \& Gong, Q. (2010). Variation in East Asian sign language structures. In Diane Brentari (Ed.), Sign languages, 502-521. Cambridge: Cambridge University Press.

Floyd, S. (2016). Modally hybrid grammar?: Celestial pointing for time-of-day reference in Nheengatú. Language, 92(1), 31-64. doi:10.1353/lan.2016.0013

Fuhrman, O., \& Boroditsky, L. (2010). Cross-cultural differences in mental representations of time: Evidence from an implicit nonlinguistic task. Cognitive Science, 34(8), 1430-1451. doi:10.1111/j.1551-6709.2010.01105.x

Fuhrman, O., McCormick, K., Chen, E., Jiang, H. D., Shu, D. F., Mao, S. M., \& Boroditsky, L. (2011). How linguistic and cultural forces shape conceptions of time: English and Mandarin time in 3D. Cognitive Science, 35(7), 1305-1328. doi:10.1111/j.1551-6709.2011.01193.x

Gentner, D., Imai, M., \& Boroditsky, L. (2002). As time goes by: Evidence for two systems in processing space time metaphors. Language and Cognitive Processes, 17(5), 537565. doi:10.1080/01690960143000317

Gibbs, R. W. (2003). Embodied experience and linguistic meaning. Brain and Language, 84(1), 1-15. doi:10.1016/s0093-934x(02)00517-5

Giezen, M., \& Emmorey, K. (2016). Language coactivation and lexical selection in bimodal bilinguals: Evidence from picture-word interference. Bilingualism: Language and Cognition, 19(2), 264-276. doi:10.1017/S1366728915000097

Gu, Y., Hoetjes, M., \& Swerts, M. (in preparation). Different hands of time: What can Mandarin-English speakers' and Dutch-English speakers' temporal gestures reveal their conceptions of time?
Gu, Y., Mol, L., Hoetjes, M., \& Swerts, M. (2017). Conceptual and lexical effects on gestures: the case of vertical spatial metaphors for time in Chinese. Language, Cognition and Neuroscience, 32(8), 1048-1063. doi:10.1080/23273798.2017.1283425

Gu, Y., Zheng, Y., \& Swerts, M. (2017). Does Mandarin spatial metaphor for time influence Chinese deaf signers' spatio-temporal reasoning? In G. Gunzelmann, A. Howes, T. Tenbrink, E. J. Davelaar (Eds). Proceedings of the 39th Annual Conference of the Cognitive Science Society (pp.445-450). Austin, TX: Cognitive Science Society.

$\mathrm{Gu}$, Y., Zheng, Y., \& Swerts, M. (in revision). Which is in front of Chinese people, Past or Future? The effect of language and culture on temporal gestures and spatial conceptions of time. Cognitive Science.

Hendricks, R., \& Boroditsky, L. (2017). New spacetime metaphors foster new non-linguistic representations. Topics in Cognitive Science, 9(3), 800-818. doi:10.1111/tops.12279.

Hostetter, A. B., \& Alibali, M. W. (2008). Visible embodiment: Gestures as simulated action. Psychonomic Bulletin \& Review, 15(3), 495-514. doi:10.3758/pbr.15.3.495

Kita, S. (2000). How representational gestures help speaking. In D. McNeill (Eds.), Language and gesture (pp. 162185). Cambridge, MA: Cambridge University Press. doi:10.1017/cbo9780511620850.011

Kita, S., Danziger, E., \& Stolz, C. (2001). Cultural specificity of spatial schemas, as manifested in spontaneous gestures. In M. Gattis (Ed.), Spatial schemas and abstract thought (pp. 115-146). Cambridge, MA: MIT Press.

Kita, S., Alibali, M. W., \& Chu, M. (2017). How do gestures influence thinking and speaking? The Gesturefor-Conceptualization Hypothesis. Psychological Review, 124(3), 245-266. doi:10.1037/rev0000059

Kita, S., \& Özyürek, A. (2003). What does cross-linguistic variation in semantic coordination of speech and gesture reveal? Evidence for an interface representation of spatial thinking and speaking. Journal of Memory and Language, 48(1), 16-32. doi:10.1016/s0749-596x(02)00505-3

Lai, V. T., \& Boroditsky, L. (2013). The immediate and chronic influence of spatio-temporal metaphors on the mental representations of time in English, Mandarin, and Mandarin-English speakers. Frontiers in Psychology, 4, 110. doi:10.3389/fpsyg.2013.00142

Lakoff, G., \& Johnson, M. (1980). Metaphors we live by. Chicago, IL: University of Chicago Press.

Lausberg, H., \& Sloetjes, H. (2009). Coding gestural behavior with the NEUROGES-ELAN system. Behavior Research Methods, 41(3), 841-849. doi:10.3758/brm.41.3.841

Le Guen, O., \& Balam, L. I. P. (2012). No metaphorical timeline in gesture and cognition among Yucatec Mayas. Frontiers in Psychology, 3. doi:10.3389/fpsyg.2012.00271

Maeder, C., \& Loncke, F. (1996). Spatial, temporal and temporological notions in French Sign Language: Comparative study of deaf and hearing subjects. Sign Language Studies, 90(1), 38-51. doi:10.1353/sls.1996.0006

Meade, G., Midgley, K., Sevcikova Sehyr, Z., Holcomb, P., \& Emmorey, K. (2017). Implicit co-activation of American Sign Language in deaf readers: An ERP study. Brain and Language, 170, 50-61. doi:10.1016/j.band1.2017.03.004 
Morford, J. P., Wilkinson, E., Villwock, A., Piñar, P., \& Kroll, J. F. (2011). When deaf signers read English: Do written words activate their sign translations? Cognition, 118 (2), 286-292. doi:10.1016/j.cognition.2010.11.006

Moore, K. E. (2011). Ego-perspective and field-based frames of reference: Temporal meanings of FRONT in Japanese, Wolof, and Aymara. Journal of Pragmatics, 43(3), 759776. doi:10.1016/j.pragma.2010.07.003

Nilsson, A. L. (2016). Embodying metaphors: Signed language interpreters at work. Cognitive Linguistics, 27(1), 35-65. doi:10.1515/cog-2015-0029

Núñez, R., Cooperrider, K., Doan, D., \& Wassmann, J. (2012). Contours of time: Topographic construals of past, present, and future in the Yupno valley of Papua New Guinea. Cognition, 124, 25-35. doi:10.1016/j.cognition.2012.03.007

Núñez, R., \& Cooperrider, K., (2013). The tangle of space and time in human cognition. Trends in Cognitive Sciences, 17(5), 220-229. doi:10.1016/j.tics.2013.03.008

Núñez, R., Motz, B., \& Teuscher, U. (2006). Time after time: The psychological reality of the ego- and timereference-point distinction in metaphorical construals of time. Metaphor and Symbol, 21(3), 133-146. doi:10.1207/s15327868ms2103_1

Núñez, R. E., \& Sweetser, E. (2006). With the future behind them: Convergent evidence from Aymara language and gesture in the crosslinguistic comparison of spatial construals of time. Cognitive Science, 30(3), 401-450. doi:10.1207/s15516709cog0000_62

Ortega, G., \& Morgan, G. (2015). The effect of sign iconicity in the mental lexicon of hearing non-signers and proficient signers: Evidence of cross-modal priming. Language, Cognition and Neuroscience, 30(5), 574-585. doi:10.1080/23273798.2014.959533.

Özçalışkan, Ş. (2016). Do gestures follow speech in bilinguals' description of motion? Bilingualism: Language and Cognition, 19(3), 644-653. doi:10.1017/s1366728915000796

Özçalışkan, Ş, Lucero, C., \& Goldin-Meadow, S. (2016). Does language shape silent gesture? Cognition, 148, 10-18. doi:10.1016/j.cognition.2015.12.001

Pika, S., Nicoladis, E., \& Marentette, P. F. (2006). A crosscultural study on the use of gestures: Evidence for crosslinguistic transfer? Bilingualism: Language and Cognition, 9(3), 319-327. doi:10.1017/S1366728906002665

Pyers, J., \& Emmorey, K. (2008). The face of bimodal bilingualism: Grammatical markers in American Sign Language are produced when bilinguals speak to English monolinguals. Psychological Science, 19(6), 531-536. doi:10.1111/j.1467-9280.2008.02119.x

Schermer, T., \& Koolhof, C. (1990). The reality of time-lines: Aspects of tense in Sign Language of the Netherlands (SLN). In S. Prillwitz \& T. Vollhaber (Eds.), Current trends in European sign language research, pp. 295-305. Hamburg: Signum.

Sinte, A. (2013). Expression of time in French Belgian Sign Language (LSFB). In Meurant, L. Sinte, A., Van
Herreweghe, M., \& Vermeerbergen, M. (Eds.), Sign language research, uses and practices: Crossing views on theoretical and applied sign language linguistics (pp. 205235). Berlin: De Gruyter Mouton and Ishara Press. doi: 10.1515/9781614511472.205

Tversky, B. (2011). Visualizing Thought. Topics in Cognitive Science, 3(3), 499-535. doi:10.1111/ j.1756-8765.2010.01113.x

Van Hell, J. G., \& Dijkstra, T. (2002). Foreign language knowledge can influence native language performance in exclusively native contexts. Psychonomic Bulletin \& Review, 9(4), 780-789. doi:10.3758/BF03196335

Walker, E. J., Bergen, B. K., \& Núñez, R. (2017). The spatial alignment of time: Differences in alignment of deictic and sequence time along the sagittal and lateral axes. Acta Psychologica, 175, 13-20. doi: 10.1016/j.actpsy.2017.02.001

Walker, E., \& Cooperrider, K. (2016). The continuity of metaphor: Evidence from temporal gestures. Cognitive Science, 40(2), 481-495. doi:10.1111/cogs. 12254

Wilcox, S. (2002). The iconic mapping of space and time in signed languages. In L. Albertazzi (Ed.), Unfolding perceptual continua, 255-281. Amsterdam \& Philadelphia: John Benjamins. doi:10.1075/aicr.41.12wil

Wu, L., \& Li, H. (2012). Time-space metaphors in Chinese Sign Language (中国手语中的时间空间隐喻). Chinese Journal of Special Education, 150(12), 2529.

Wu, Y. J., \& Thierry, G. (2010). Chinese-English bilinguals reading English hear Chinese. Journal of Neuroscience, 30(22), 7646-7651. doi:10.1523/jneurosci.1602-10.2010

Xiao, C., Zhao, M., \& Chen, L. (2017). Both earlier times and the future are "Front": The distinction between time- and ego-reference-points in Mandarin speakers' temporal representation. Cognitive Science, 42(3), 10261040. doi: $10.1111 / \operatorname{cogs} .12552$

Yang, J. H. (2005). Expressing negation in Chinese sign language. MA Thesis. Rochester Institute of Technology. Accessed from http://scholarworks.rit.edu/theses/4010

Yang, J. H. (2015) Chinese Sign Language. In Jepsen, J.B., De Clerck, G., Lutalo-Kiingi, S., McGregor, W. B. (eds), Sign languages of the world: $A$ comparative handbook (pp. 177-194). Berlin: De Gruyter. doi:10.1515/9781614518174-012

$\mathrm{Yu}, \mathrm{N}$. (2012). The metaphorical orientation of time in Chinese. Journal of Pragmatics, 44(10), 1335-1354. doi:10.1016/j.pragma.2012.06.002

Zheng, X. (2009). A study of signs for non-visual concepts in Shanghai Variety of Chinese Sign Language (上海手语非 视觉概念表达研究). PhD Dissertation. Fudan University, China.

Zou, L., Abutalebi, J., Zinszer, B., Yan, X., Shu, H., Peng, D., \& Ding, G. (2012). Second language experience modulates functional brain network for the native language production in bimodal bilinguals. NeuroImage, 62(3), 1367-1375. doi:10.1016/j.neuroimage.2012.05.062 